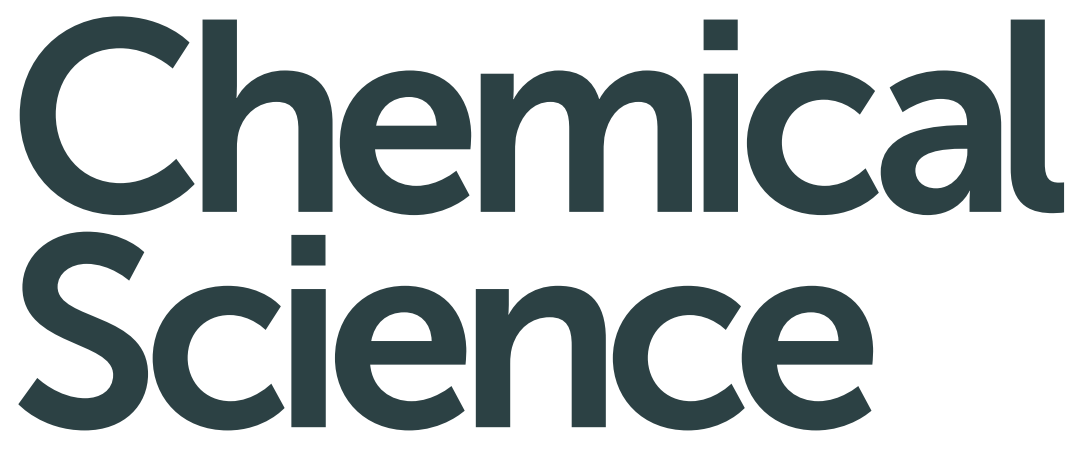

rsc.li/chemical-science

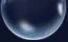
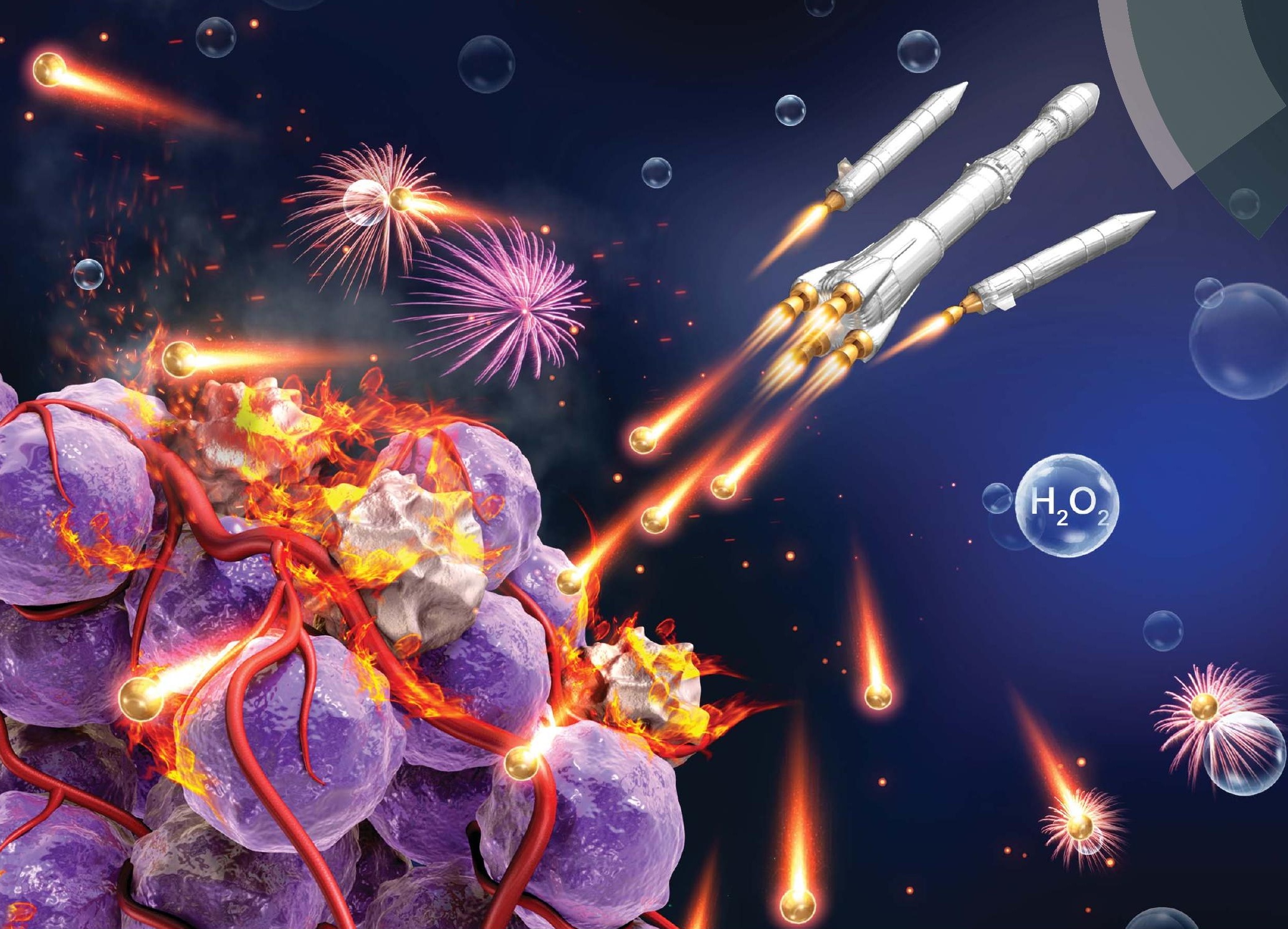

ISSN 2041-6539

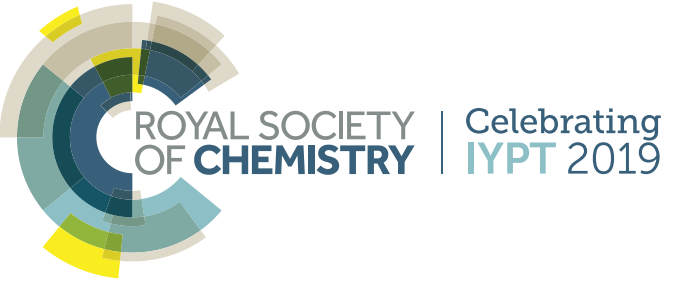

EDGE ARTICLE

Yan Ma, Yang Lu, Zhengbao Zha et al.

PEGylated rhenium nanoclusters: a degradable metal

photothermal nanoagent for cancer therapy 
Check for updates

Cite this: Chem. Sci., 2019, 10, 5435

๑ All publication charges for this article have been paid for by the Royal Society of Chemistry

Received 12th February 2019

Accepted 22nd April 2019

DOI: $10.1039 / \mathrm{c} 9 \mathrm{sc} 00729 f$

rsc.li/chemical-science

\section{PEGylated rhenium nanoclusters: a degradable metal photothermal nanoagent for cancer therapy $\dagger$}

\author{
Zhaohua Miao, $\$^{\mathrm{ab}}$ Sheng Chen, $\$^{\mathrm{c}}$ Cheng-Yan Xu, (D) ${ }^{\mathrm{b}}$ Yan Ma, ${ }^{\text {*a }}$ Haisheng Qian, (D) ${ }^{a}$
} Yunjun $\mathrm{Xu},{ }^{d}$ Huajian Chen, ${ }^{a}$ Xianwen Wang, ${ }^{a}$ Gang He, ${ }^{a}$ Yang Lu, (DD *c Qingliang Zhao and Zhengbao Zha $\mathbb{D}$ *a

\begin{abstract}
A common issue of functional nanoagents for potential clinical translation is whether they are biodegradable or renal clearable. Previous studies have widely explored noble metal nanoparticles (Au and $\mathrm{Pd}$ ) as the first generation of photothermal nanoagents for cancer therapy, but all of the reported noble metal nanoparticles are non-degradable. On the other hand, rhenium (Re), one of the noble and precious metals with a high atomic number $(Z=75)$, has been mainly utilized as a jet superalloy or chemical catalyst, but the biological characteristics and activity of Re nanoparticles have never been evaluated until now. To address these issues, here we report a simple and scalable liquid-reduction strategy to synthesize PEGylated Re nanoclusters, which exhibit intrinsically high photothermal conversion efficacy $(33.0 \%)$ and high $\mathrm{X}$-ray attenuation $\left(21.2 \mathrm{HU} \mathrm{mL} \mathrm{mg}{ }^{-1}\right.$ ), resulting in excellent photothermal ablation (100\% tumor elimination) and higher CT enhancement $\left(15.9 \mathrm{HU} \mathrm{mL} \mathrm{mg}{ }^{-1}\right.$ for commercial iopromide in clinics). Impressively, biocompatible Re nanoclusters can degrade into renal clearable $\mathrm{ReO}_{4}{ }^{-}$ions after exposure to $\mathrm{H}_{2} \mathrm{O}_{2}$, and thus achieve much higher renal clearance efficiency than conventional gold nanoparticles. This work reveals the potential of theranostic application of metallic Re nanoclusters with both biodegradation and renal clearance properties and provides insights into the design of degradable metallic platforms with high clinical prospects.
\end{abstract}

\section{Introduction}

Commonly, most nanoagents suitable for potential clinical applications of diagnosis and treatment are likely to have either ultrasmall size or degradability, in order to meet the demand of complete clearance from the body within a reasonable period of time..$^{1-3}$ It is generally accepted that ultrasmall nanoparticles with hydrodynamic diameters less than $8 \mathrm{~nm}$ can undergo renal clearance through effective glomerulus filtration. ${ }^{4-6}$ Until now, numerous ultrasmall nanomaterials with strong near-infrared (NIR) absorption including polyoxometalate clusters, ${ }^{2} \mathrm{CuS}$

${ }^{a}$ School of Food and Biological Engineering, Hefei University of Technology, Hefei, Anhui 230009, P. R. China. E-mail: yanma@hfut.edu.cn; zbzha@hfut.edu.cn

${ }^{b}$ State Key Laboratory of Advanced Welding and Joining, Harbin Institute of Technology, Harbin, 150001, P. R. China

${ }^{c}$ School of Chemistry and Chemical Engineering, Hefei University of Technology, Hefei, Anhui, 230009, P. R. China. E-mail: yanglu@hfut.edu.cn

${ }^{d}$ The First Affiliated Hospital of University of Science and Technology of China, Anhui Province Hospital, Hefei 230001, P. R. China

${ }^{e}$ State Key Laboratory of Molecular Vaccinology and Molecular Diagnostics, Center for Molecular Imaging and Translational Medicine, School of Public Health, Xiamen University, Xiamen 361102, China

$\dagger$ Electronic supplementary information (ESI) available: Photothermal conversion efficiency calculation, XPS spectra of Re nanoclusters, digital photographs and H\&E images of major organs. See DOI: 10.1039/c9sc00729f

$\$$ These authors contributed equally to this work. nanodots, ${ }^{7}$ bismuth nanodots ${ }^{8}$ and platinum nanodots ${ }^{9}$ have been explored for cancer diagnosis and photothermal therapy (PTT), a potential alternative to surgery due to the merits of noninvasiveness, local laser-targeting, quick recovery and easy operation..$^{10-15}$ However, considering the complex environment in vivo (serum protein coating, phagocytic cell uptake, etc.), the long-term retention of these nanoparticles in the body is still possible. ${ }^{16-18}$ Hence, much attention has been paid to the construction of nanoparticles with both biodegradation and renal clearance properties, which mainly focused on $\mathrm{Fe}^{3+}$-gallic acid nanodots, ${ }^{1}$ porphyrin nanodots ${ }^{19}$ and black phosphorus. ${ }^{20}$ Especially, black phosphorus quantum dots have recently captivated many researchers for the inherent feature of being oxidized into biological endogenous $\mathrm{PO}_{4}{ }^{3-}$, resulting in the potential of perfect biodegradability in vivo. ${ }^{21-24}$ However, the fabrication of black phosphorus quantum dots is timeconsuming and expensive. ${ }^{21-24}$ In addition, although noble metal nanoparticles including Au nanoshells, ${ }^{25} \mathrm{Au}$ nanorods $\mathrm{s}^{26,27}$ and Pd nanosheets ${ }^{28}$ as the first generation of photothermal agents have been widely reported for a long time, there are still no reports of biodegradable noble metal nanoparticles for PTT because of the inertness and non-biodegradability of noble metals, which have led to the risk of potential long-term retention toxicity in vivo and may hamper their further clinical translation efforts. ${ }^{29,30}$ Hence, it is of urgency and importance to 
develop degradable noble metal nanoparticles to satisfy the demands of clinical translation.

In the transition-metal group of the periodic table, rhenium $(\mathrm{Re})$ is a rare and powerful noble metal with a high atomic number $(Z=75)$. Bulk Re materials have excellent corrosion resistance and are widely utilized as a superalloy of jet engines, while Re nanomaterials are easily oxidized after exposure to oxygen, indicating the potential of biodegradation, and can also serve as a chemical nanocatalyst. ${ }^{31-33}$ However, to date, there are no reports of the biological characteristics and activity of Re nanoparticles and their toxicity has never been evaluated. Fortunately, several rhenium compounds (i.e. ${ }^{188}$ Re-hydroxyethylidene diphosphonate) have been explored for PET imaging or radiotherapy in clinical trials, ${ }^{34,35}$ and our group has also demonstrated that liquid exfoliated $\mathrm{ReS}_{2}$ nanosheets could serve as multifunctional theranostic agents which are still unbiodegradable. ${ }^{36}$ In addition, the lethal dose $50 \%\left(\mathrm{LD}_{50}\right)$ of $\mathrm{KReO}_{4}$ is reported to be as high as $2800 \mathrm{mg} \mathrm{kg}{ }^{-1},{ }^{37}$ indicating that $\mathrm{ReO}_{4}{ }^{-}$ions, a typical oxidization product of Re nanoparticles, should be biocompatible. Therefore, the exploration of biomedical applications of Re nanomaterials is intriguing.

Herein, inspired by the degradation of black phosphorus into $\mathrm{PO}_{4}{ }^{3-}$ upon exposure to oxygen, we report a simple and scalable one-step strategy to synthesize PEGylated rhenium nanoclusters (Re NCs) as degradable noble metallic nanoparticles for biomedical applications (Scheme 1a). Owing to relatively strong absorbance in the NIR region, Re NCs can effectively ablate tumors through significant photothermal effects. In addition, similar to gold nanostructures, Re nanoclusters can also serve as excellent CT contrast agents because of the presence of the high atomic number element Re (Scheme 1b). However, significantly different from other noble metal nanoparticles, Re NCs can be easily transformed into biocompatible $\mathrm{ReO}_{4}{ }^{-}$ions after exposure to $\mathrm{H}_{2} \mathrm{O}_{2}$ (Scheme 1c), ensuring the potential of biodegradability in the body especially for tumors because the concentration of endogenous $\mathrm{H}_{2} \mathrm{O}_{2}$ inside most types of solid tumors is in the range of 10$100 \mu \mathrm{M}$, much higher than that of normal tissues. ${ }^{38-40} \mathrm{~A}$ further

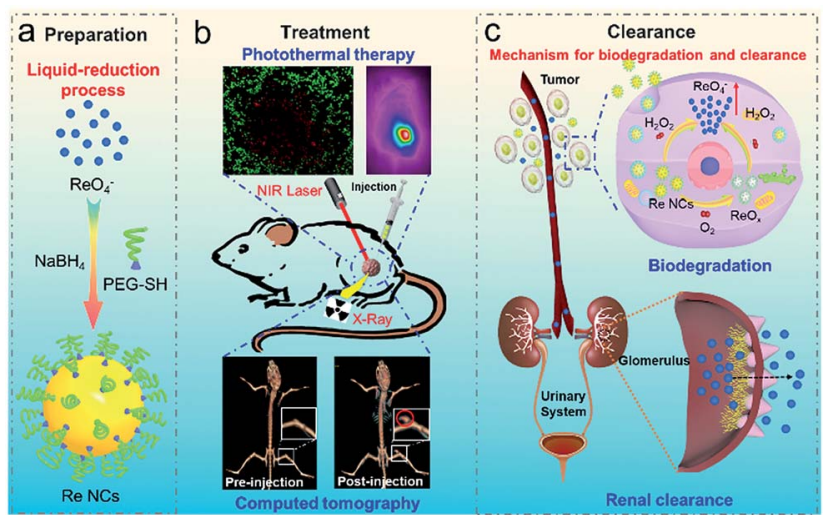

Scheme 1 Schematic of degradable and renal-clearable PEGylated rhenium nanoclusters for tumor diagnosis and therapy. (a) Preparation procedure of Re NCs; (b) PTT and CT imaging; (c) mechanism for the biodegradation renal clearance process of Re NCs. renal-clearance experiment reveals that Re NCs exhibit similar renal-clearance properties to soluble $\mathrm{NH}_{4} \mathrm{ReO}_{4}$, but much higher renal clearance efficiency than conventional gold nanoparticles with similar sizes and modifications, indicating high potential of complete clearance from the body in a reasonable time period. The obtained Re nanoclusters represent a relatively ideal theranostic agent that integrates the merits of two outstanding "state of the art" photothermal nanoagents (i.e. gold nanostructures and black phosphorus). Benefiting from the features of strong NIR absorption and high X-ray attenuation like gold nanostructures, and stimuliresponsive degradation and ultrasmall size $(\sim 2 \mathrm{~nm})$ like black phosphorus quantum dots, multifunctional Re NCs as a new generation of photothermal agents are believed to hold great potential for clinical translation. To the best of our knowledge, this is the first report about biodegradable and renal-clearable noble metallic theranostic nanoagents for CT imaging and PTT.

\section{Experimental section}

\section{Reagents and chemicals}

Ammonium perrhenate $\left(\mathrm{NH}_{4} \mathrm{ReO}_{4}\right)$ was purchased from SigmaAldrich. PEG-SH was obtained from Shanghai YareBio Company. Sodium borohydride was supplied by Sinopharm Chemical Reagent Beijing Co., Ltd. D.I. water used in this experiment was acquired using a Milli-Q water purification system from Millipore.

\section{Synthesis of rhenium nanoclusters}

Typically, $100 \mathrm{mg}$ ammonium perrhenate and $45 \mathrm{mg}$ PEG-SH were added into $25 \mathrm{~mL}$ of D.I. water, followed by the dropwise addition of $5 \mathrm{~mL}$ of $\mathrm{NaBH}_{4}$ solution $\left(30 \mathrm{mg} \mathrm{mL}{ }^{-1}\right)$. After vigorous stirring for $2 \mathrm{~h}$, the color of the above mixed solution changed from colorless to dark brown. The ultrasmall Re nanoclusters were obtained by ultrafiltration centrifugation (4500 rpm, $10 \mathrm{~min}$ ) and washed with D.I. water four times.

\section{Characterization}

The morphologies of PEGylated rhenium nanoclusters were observed by using a transmission electron microscope (TEM, JEOL JEM-2100F). X-ray photoelectron spectroscopy (XPS) spectra were recorded on an X-ray photoelectron spectrometer (ESCALab 250Xi). UV-vis-NIR absorption spectra were measured by using a Hitachi U-5100 spectrophotometer. The zeta potential and dynamic light scattering (DLS) particle size were acquired by using a PALS/90Plus instrument (Brookhaven). The Fourier transform infrared spectroscopy (FTIR) spectra of Re nanoclusters and PEG-SH were measured by using a Varian 3000 FTIR spectrophotometer. The rhenium element concentration before and after dialysis was measured with an AA800 Atomic Absorption Spectrometer.

\section{Photothermal effect of rhenium nanoclusters}

$2 \mathrm{~mL}$ of Re nanocluster aqueous dispersion with different concentrations were stored in a quartz cuvette, and then 
illuminated with an $808 \mathrm{~nm}$ laser (2 W) for $10 \mathrm{~min}$. D.I. water was used as a control. A thermocouple probe with a digital thermometer was used to record the temperature of aqueous dispersions at different time points. The detailed calculation procedure of photothermal conversion efficacy is provided in the ESI. $\dagger$

\section{Storage stability of Re nanoclusters}

To evaluate the stability, Re nanocluster aqueous dispersions were stored at four different temperatures $(-20,4,25$ and 37 ${ }^{\circ} \mathrm{C}$ ), and the absorbance of these dispersions was recorded at

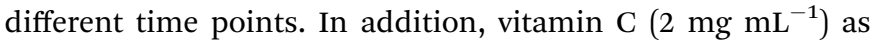
a reducing protective agent was added in another group, and the absorbance change was also recorded.

\section{$\mathrm{H}_{2} \mathrm{O}_{2}$-responsive degradability}

Re nanoclusters were dispersed in different concentrations of $\mathrm{H}_{2} \mathrm{O}_{2}$ solutions $(0,0.25 \%, 0.5 \%$ and $1 \%)$ to modulate tumor microenvironments with a high concentration of $\mathrm{H}_{2} \mathrm{O}_{2}$ for $24 \mathrm{~h}$, and then transferred into a dialysis bag and dialyzed in D.I. water for 2 days. The concentrations of Re element in the dialysis bags before and after dialysis were measured by ICP-AES. The morphologies of the samples before and after dialysis were observed by TEM, and XPS was also conducted to identify the valence states of Re element.

\section{Cytotoxicity assay}

Typical methyl thiazolyl tetrazolium (MTT) assay was performed to study the cytotoxicity of Re nanoclusters using HUVECs as model cells. The cells with a density of $\sim 1 \times 10^{4}$ cells per well were pre-seeded in a 96-well plate and were incubated in a cell culture medium with different concentrations of Re nanoclusters $(0,15,30,60,125,250$ and $500 \mathrm{ppm})$ for $24 \mathrm{~h}$. Then, 20

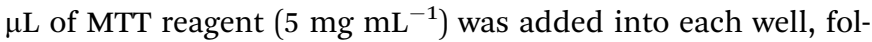
lowed by further incubation for $4 \mathrm{~h}$. Upon the addition of DMSO $(150 \mu \mathrm{L})$ to dissolve the formazan crystals, the relative cell viability was determined based on the absorbance of the formazan product at $570 \mathrm{~nm}$ using a microplate reader. The same process was adopted for the cytotoxicity evaluation of ammonium perrhenate and the oxidation product of Re nanoclusters.

\section{In vitro photothermal ablation assay}

To evaluate the photothermal cytotoxicity of Re nanoclusters, 4T1 cells were incubated with different concentrations of Re nanocluster dispersions $(0,7.8,15.6,31.2,65,125$ and $250 \mathrm{ppm})$ in a 96-well plate, and then irradiated with the $808 \mathrm{~nm}$ laser $(2$ W, $5 \mathrm{~min}$ ) with an irradiation area of $\sim 0.33 \mathrm{~cm}^{2}$. The cell viabilities after laser irradiation were determined by the standard MTT assay described above. In addition, 4T1 cells were incubated with Re nanocluster suspensions with different concentrations for $4 \mathrm{~h}$ in a 6-well plate, and irradiated with the $808 \mathrm{~nm}$ laser ( $2 \mathrm{~W}, 5 \mathrm{~min}$ ). After the irradiation, the cells were stained with calcein acetoxymethyl ester and propidium iodide for the visualization of live and dead cells, respectively.

\section{Animal model}

BALB/c mice were used as model animals. The tumor model was established through subcutaneous injection of 4 T1 cells $(\sim 1 \times$ $10^{6}$ in $100 \mu \mathrm{L}$ PBS solution) into the back section of BALB/c mice. The mice were used when the tumor volume of each mouse reached about $30 \mathrm{~mm}^{3}$. All animal procedures were performed in accordance with the Guidelines for Care and Use of Laboratory Animals of Anhui Medical University and experiments were approved by the Institutional Animal Care and Use Committee of Anhui Medical University.

\section{Pharmacokinetic and biodistribution analysis in vivo}

For blood circulation and biodistribution, healthy ICR mice as model mice were intravenously injected with $0.1 \mathrm{~mL}$ of Re NCs ( $2 \mathrm{mg} \mathrm{mL} \mathrm{m}^{-1}$ ). Blood and major organs (heart, liver, spleen, lung and kidney) were then extracted from mice at different time points, followed by the decomposition with digestion apparatus. Finally, the Re concentration was measured by ICP-AES (Optima 7300 DV).

For renal clearance, twelve mice were randomly divided into three groups: (1) Re NCs, (2) $\mathrm{NH}_{4} \mathrm{ReO}_{4}$, and (3) Au nanoparticles. $0.1 \mathrm{~mL}$ of $\mathrm{Re} \mathrm{NCs}$ or $\mathrm{NH}_{4} \mathrm{ReO}_{4}$ or Au nanoparticles $\left(2 \mathrm{mg} \mathrm{mL}{ }^{-1}\right)$ was intravenously injected into the mice in metabolic cages. The urine samples from each mouse were collected at $24 \mathrm{~h}$ after administration, and the concentration of Re element in urine was determined by using an AA800 Atomic Absorption Spectrometer.

\section{In vivo photothermal ablation assay}

Twenty 4T1 tumor-bearing mice were randomly divided into four different groups ( $n=5$, in each group) as follows: (1) PBS, (2) Re, (3) PBS + NIR and (4) Re + NIR. The mice from groups 2 and 4 were intratumorally injected with $\mathrm{Re}$ nanocluster dispersions $\left(25 \mu \mathrm{L}, 2 \mathrm{mg} \mathrm{mL}^{-1}\right)$. After the mice were anesthetized, the tumors were irradiated with a NIR laser $(808 \mathrm{~nm}, 1$ $\mathrm{W} \mathrm{cm}^{-2}$ ) for $10 \mathrm{~min}$. During the treatment, an infrared thermal camera (Ti400, Fluke, USA) was used to monitor the temperature change of the tumor site. After laser irradiation, the tumor volumes and body weights of all mice were measured every two days using an electronic balance and a caliper. The tumor volume was calculated by using the formula: $V=a b^{2} / 2$ ( $a$ for length and $b$ for width). After 16 days, the tumors were dissected and weighed to evaluate the therapeutic efficacy, and major organs (heart, liver, spleen, lung and kidney) from the "Re + NIR" group were stained for histology analysis.

\section{CT imaging}

To evaluate the CT contrast efficacy, in vitro and in vivo CT imaging experiments were conducted. For in vitro imaging, Re nanocluster dispersions at different concentrations $(0,0.75,1.5$, 3 , 6 and $12 \mathrm{mg} \mathrm{mL}^{-1}$ ) were measured by using a clinical CT scanner (GE, Discovery CT750HD, GE Healthcare, WI) with a 5 mm slice thickness at $120 \mathrm{kV}_{\mathrm{p}}$, and the CT imaging and Hounsfield unit values of each sample were recorded and calculated. To conduct in vivo CT imaging, Re nanocluster 
suspensions ( $50 \mu \mathrm{L}, 20 \mathrm{mg} \mathrm{mL} \mathrm{m}^{-1}$ ) were intratumorally administrated into 4T1 tumor-bearing mice, and the CT images of each mouse were collected and reconstructed by using the same clinical CT scanner.

\section{Statistical analysis}

All statistical analyses were performed using Excel software through two-tailed Student's $t$-test.

\section{Results and discussion}

\section{Synthesis and characterization of PEGylated Re NCs}

Re NCs are synthesized via the liquid-phase reduction of $\mathrm{NH}_{4} \mathrm{ReO}_{4}$ with $\mathrm{NaBH}_{4}$ using biocompatible PEG-SH as a surface stabilizer in an aqueous environment. Transmission electron microscope (TEM) images reveal that the obtained Re NCs are irregular sphere particles with diameters ranging from 1 to 2 $\mathrm{nm}$ due to partial oxidation upon exposure to air (Fig. 1a and S1†). High-resolution transmission electron microscopy (HRTEM) depicts an interplanar spacing of $2.39 \AA$ A, consistent with (100) crystallographic planes in a hexagonal structure of Re (Fig. S2 $\dagger$ ). ${ }^{33}$ The hydrodynamic diameter (Fig. 1b) of Re NCs is $\sim 7.6 \mathrm{~nm}$ in deionized water, larger than the diameter of NCs determined by TEM, which should be mainly ascribed to the low contrast of PEG-SH by TEM and the surrounding water shells. Fourier transform infrared spectra (Fig. 1c) were then recorded, and the characteristic peaks of PEG-SH molecules at $2922 \mathrm{~cm}^{-1}$, $2877 \mathrm{~cm}^{-1}$ (alkyl C-H stretching) and $1099.2 \mathrm{~cm}^{-1}$ (C-O-C stretching) are observed for PEGylated Re NCs, confirming the successful conjugation of biocompatible PEG-SH on the surface of Re NCs. Interestingly, when PEG-SH is replaced with PEG$\mathrm{NH}_{2}$ or DSPE-PEG, the obtained Re NCs easily aggregate. The possible reason is the formation of a unique Re-S bond between Re and PEG-SH like an Au-S covalent bond (inset of Fig. 1d), while the force between Re NCs and PEG-NH $\mathrm{N}_{2}$ or DSPE-PEG is weak. ${ }^{41}$ The bonding between Re and $\mathrm{S}$ reveals a facile strategy to modify Re nanomaterials for biomedical applications and may promote their application in biomedical fields.

To monitor the reduction procedure, the absorbance spectra of reaction solution at different time points are measured (Fig. 1d). It is found that the absorbance of reaction solution after $2 \mathrm{~h}$ reaches equilibrium, indicating that the reduction of $\mathrm{NH}_{4} \mathrm{ReO}_{4}$ can be accomplished in $2 \mathrm{~h}$. The obtained Re NCs show no surface plasmon resonance (SPR) maximum probably due to the low dielectric constant, ${ }^{42}$ but have obvious absorbance from the UV to the NIR region (Fig. 1e and S3 $†$ ). The inset in Fig. $\mathrm{S} 3 \dagger$ is the digital image of Re NC dispersions at different concentrations, indicating the good dispersion of Re NCs in water due to the modification of PEG-SH. The mass extinction coefficient of Re NCs is determined to be $2.6 \mathrm{~L} \mathrm{~g}^{-1} \mathrm{~cm}^{-1}$ at 808 $\mathrm{nm}$ based on the Lambert-Beer law $(A / L=\alpha C, \alpha$ represents the mass extinction coefficient) and compared to that of graphene oxide $\left(3.6 \mathrm{~L} \mathrm{~g}^{-1} \mathrm{~cm}^{-1}\right) .{ }^{20}$ Inspired by the reasonable absorption in the NIR region, the photothermal performance of Re NCs is evaluated. As shown in Fig. 1f, the photothermal heating curves of Re NCs demonstrate that the temperature change is
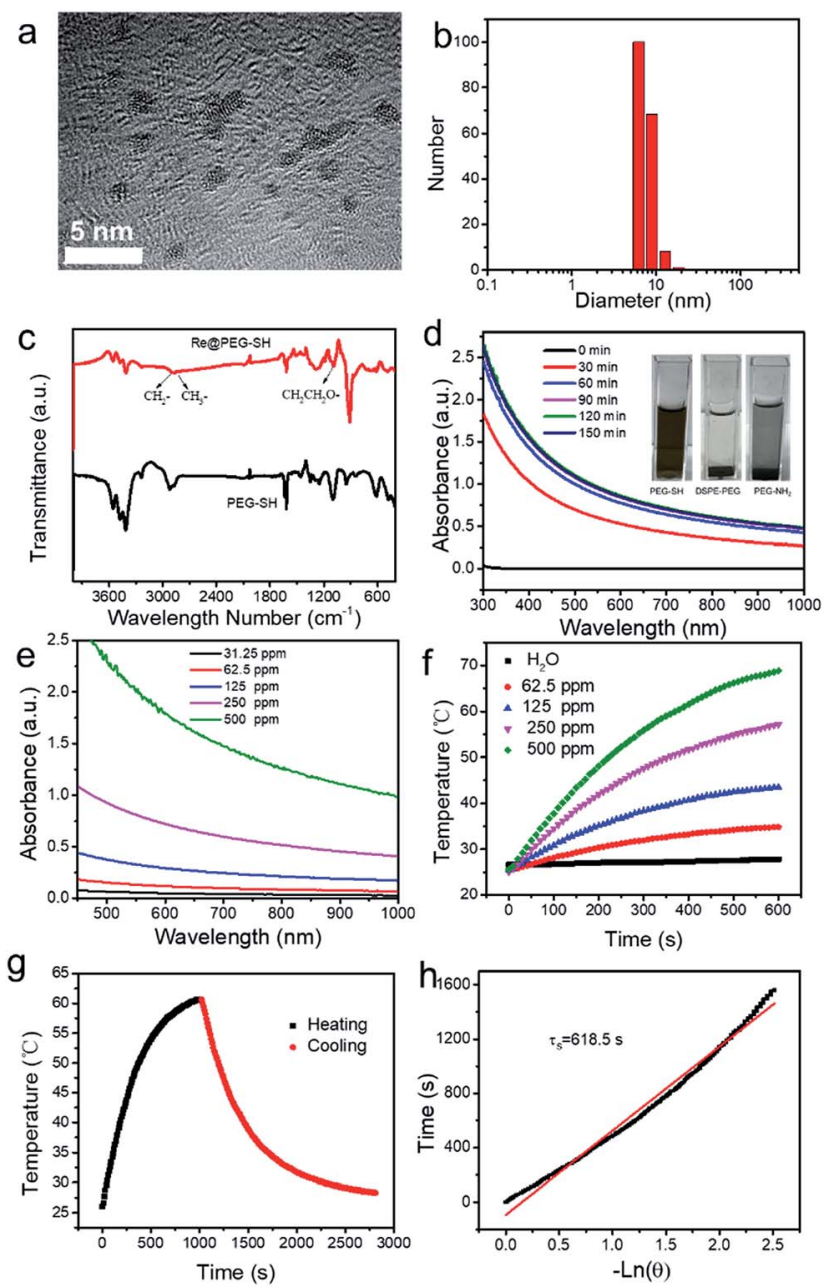

Fig. 1 Fabrication and characterization of Re NCs. (a) TEM image; (b) DLS distribution; (c) FTIR spectra of Re NCs and PEG-SH; (d) UV-visNIR absorption at different time points during fabrication; inset is a digital image of Re NC dispersions modified with different kinds of PEG; (e) UV-vis-NIR absorption of Re NC dispersions at different concentrations; (f) photothermal heating curves of Re NC dispersions at different concentrations and pure water; $(\mathrm{g})$ the heating/cooling curves of Re NCs (250 ppm) under laser irradiation (2 W, $808 \mathrm{~nm}$ ); (h) the fitting linear curve of time data vs. $-\ln \theta$ from the cooling period of Re NCs.

positively associated with irradiation time and concentrations. For example, the temperature of the Re NC dispersion with a concentration of $250 \mathrm{ppm}$ raises from 25 to $57.1^{\circ} \mathrm{C}$ under laser irradiation $(2 \mathrm{~W}, 808 \mathrm{~nm})$. The photothermal conversion efficacy (PCE) of Re NCs is determined to be $33.0 \%$ (Fig. 1g and h), higher than those of black phosphorus quantum dots $(28.4 \%)^{20}$ and golden nanorods (21\%). For direct comparison, the PTCE of exfoliated black phosphorus was calculated to be $26.7 \%$ under the same conditions (Fig. S4†), indicating a relatively high PTCE of Re NCs. In addition, Re NCs have better photothermal stability than golden nanorods although a little temperature decrease is observed, which should be attributed to the partial oxidation of Re NCs during irradiation (Fig. S5 $\dagger$ ). Hence, relatively high PCE and photothermal stability indicate that Re NCs can serve as highly efficient photothermal agents. 


\section{$\mathrm{H}_{2} \mathrm{O}_{2}$-responsive degradability and storage stability of Re NCs}

The biggest issue for metal nanomaterials (i.e. Au and Pd) to be employed for clinical applications is their non-biodegradability, resulting in the concern of long-term toxicity in vivo. Fortunately, it has been reported that Re nanoparticles are oxidized after exposure to oxygen. ${ }^{31-33}$ Thus, we predict that Re NCs may serve as biodegradable metal photothermal agents because $\mathrm{O}_{2}$ or $\mathrm{H}_{2} \mathrm{O}_{2}$ is widely present in human tissues. To confirm this hypothesis, Re NC dispersions with a concentration of $500 \mathrm{ppm}$ were incubated with deionized water, $0.25 \%, 0.5 \%$ and $1 \% \mathrm{H}_{2} \mathrm{O}_{2}$ solution, respectively. After incubation for $24 \mathrm{~h}$, the colors of Re NC dispersions incubated in $\mathrm{H}_{2} \mathrm{O}_{2}$ solution change from dark brown to nearly colorless, while a light brown color is still observed for Re NC dispersions incubated in distilled $\mathrm{H}_{2} \mathrm{O}$ (inset of Fig. 2d and e and S6 $\dagger$ ). Then, the oxidized dispersions were packaged in dialysis bags and dialyzed in D.I. water for 2 days. The concentrations of Re element in the dialysis bags before and after dialysis are measured by inductively coupled plasma atomic emission spectroscopy (ICP-AES). Impressively, the $\mathrm{Re}$ concentrations of all three dispersions incubated in $\mathrm{H}_{2} \mathrm{O}_{2}$ are almost zero while the concentrations of Re element before and after dialysis for dispersions incubated in $\mathrm{H}_{2} \mathrm{O}$ are almost the same (Fig. 2c). TEM images reveal that no visible nanostructures are observed for Re dispersions in $\mathrm{H}_{2} \mathrm{O}_{2}$ while connected nanoparticles are observed for Re dispersions in $\mathrm{H}_{2} \mathrm{O}$ (Fig. 2d and e). In addition, the absorbance of Re dispersions incubated in $\mathrm{H}_{2} \mathrm{O}$ and $\mathrm{H}_{2} \mathrm{O}_{2}$ is measured (Fig. 2b). Almost no absorbance of Re dispersions in the vis-NIR region is observed
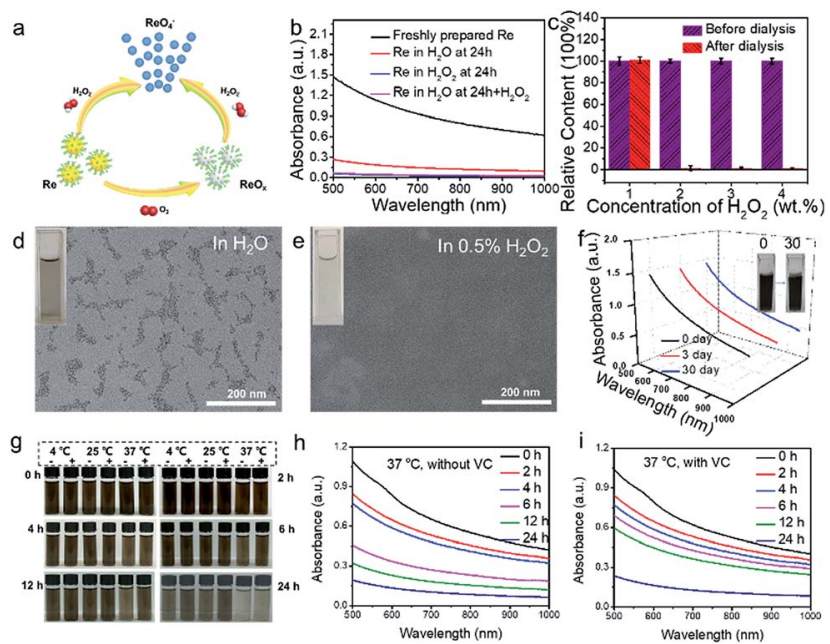

Fig. $2 \mathrm{H}_{2} \mathrm{O}_{2}$-triggered degradation and storage stability of Re NCs. (a) Schematic of $\mathrm{H}_{2} \mathrm{O}_{2}$-triggered degradation. (b) Vis-NIR absorbance of Re dispersions treated with $\mathrm{H}_{2} \mathrm{O}$ or $\mathrm{H}_{2} \mathrm{O}_{2}$. (c) Relative Re content before and after dialysis. TEM images of the degradation product of Re NCs treated with $\mathrm{H}_{2} \mathrm{O}$ (d) or $\mathrm{H}_{2} \mathrm{O}_{2}$ (e). (f) Absorbance of Re NC dispersions stored at $-20{ }^{\circ} \mathrm{C}$ for different time periods. Inset is the digital photograph of Re NC dispersions on the $0^{\text {th }}$ and the $30^{\text {th }}$ day. $(\mathrm{g})$ Stability of Re nanocluster dispersions with (+) or without (-) vitamin C at different temperatures $\left(4,25\right.$, and $37^{\circ} \mathrm{C}$ ) for different time periods. (h and i) The corresponding vis-NIR absorbance of Re nanocluster dispersions with or without vitamin $\mathrm{C}$ at $37{ }^{\circ} \mathrm{C}$ for different time periods. after the exposure to $\mathrm{H}_{2} \mathrm{O}_{2}$ while a decreased absorbance of Re dispersions incubated in $\mathrm{H}_{2} \mathrm{O}$ is observed, which also becomes nearly zero after the addition of external $\mathrm{H}_{2} \mathrm{O}_{2}$. These results suggest that the Re oxidation in $\mathrm{H}_{2} \mathrm{O}$ and $\mathrm{H}_{2} \mathrm{O}_{2}$ are two different processes, and the possible degradation mechanism is proposed in Fig. 2a. Zero-valent $\operatorname{Re}(0)$ can be transformed into $\mathrm{ReO}_{4}{ }^{-}$ions under the treatment of $\mathrm{H}_{2} \mathrm{O}_{2},{ }^{31}$ resulting in the escape from dialysis bags and no visible nanostructures by TEM (Fig. 2e). However, when incubated with $\mathrm{H}_{2} \mathrm{O}$, Re NCs react with dissolved $\mathrm{O}_{2}$ in $\mathrm{H}_{2} \mathrm{O}$ and may only be oxidized to rhenium oxide $\left(\mathrm{ReO}_{x}\right)$ which is still in the form of nanoparticles (Fig. 2d), ${ }^{33}$ attributed to the poorer oxidation capacity of $\mathrm{O}_{2}$ than $\mathrm{H}_{2} \mathrm{O}_{2}$. In addition, $\mathrm{ReO}_{x}$ nanoparticles are likely to be transformed into $\mathrm{ReO}_{4}{ }^{-}$ions after the exposure to $\mathrm{H}_{2} \mathrm{O}_{2}$. To further demonstrate the oxidation, X-ray photoelectron spectroscopy (XPS) is conducted to identify the valence states of Re NCs. Compared with freshly prepared Re NCs, a higher valence state of Re element is observed for Re NCs stored in $\mathrm{H}_{2} \mathrm{O}_{2}(0.5 \%)$ for $12 \mathrm{~h}$, which should be attributed to the transformation of $\mathrm{ReO}_{4}{ }^{-}$from $\operatorname{Re}(0)$ after exposure to $\mathrm{H}_{2} \mathrm{O}_{2}$ (Fig. $\mathrm{S} 7 \dagger$ ). ${ }^{31}$ Hence, the mechanism of Re NCs should be attributed to the oxidation-reduction reaction, in which metal Re NCs are oxidized to soluble $\mathrm{ReO}_{4}{ }^{-}$for further renal clearance when exposed to $\mathrm{H}_{2} \mathrm{O}_{2}$. Such degradation behavior is similar to that of black phosphorus quantum dots, which can be oxidized into biocompatible $\mathrm{PO}_{4}{ }^{3-}$, but is different from the disassembly of PEGylated self-assembled molecules. ${ }^{43}$ Hence, Re NCs with $\mathrm{H}_{2} \mathrm{O}_{2}$-triggered degradation behavior may have higher clinical prospects than traditional gold nanomaterials.

Considering the oxidation feature of Re NCs for further in vivo application, the stability of aqueous dispersions at different temperature $\left(4,25\right.$ and $\left.37^{\circ} \mathrm{C}\right)$ is evaluated. As shown in Fig. $2 \mathrm{~g}$, the oxidation process is accelerated with increasing temperature and the absorbance of Re NCs can remain at $\sim 75 \%$ that of the original dispersion after incubation for $4 \mathrm{~h}$ at $37{ }^{\circ} \mathrm{C}$, and such a period is long enough for photothermal ablation of tumors in vivo. However, after incubation for $24 \mathrm{~h}$, the absorbance significantly decreased to be $\sim 15 \%$, indicating that the majority of Re NCs have been oxidized. In addition, we find that the stability of Re NCs can be obviously enhanced after the addition of vitamin $\mathrm{C}$ (Fig. $2 \mathrm{~h}$ and $\mathrm{i}$ ), suggesting that Re NCs could be protected by reducing agents to control the oxidization process. Excitingly, when the Re NCs are stored at $-20{ }^{\circ} \mathrm{C}$ for even one month, no absorbance decrease of Re NCs is observed because no oxygen can come into contact with Re NCs after the formation of ice from water, ensuring the potential of long-term storage for practical application (Fig. 2f). For further biomedical applications, the storage stability of Re NCs can be enhanced by incorporation into poly(lactic-co-glycolic acid) (PLGA) or liposome nanoparticles to reduce the contact with oxygen as shown in some previous reports. ${ }^{\mathbf{1 2 , 4 0 , 4 4}}$

\section{Pharmacokinetic and biodistribution analysis of Re NCs}

Prior to biomedical applications, the blood circulation and biodistribution of Re NCs are examined by the intravenous injection of $0.1 \mathrm{~mL}$ of $\mathrm{Re} \mathrm{NC}$ dispersions $\left(2 \mathrm{mg} \mathrm{mL}^{-1}\right)$ into 
healthy ICR mice, followed by measuring the Re concentration in blood and major organs (heart, liver, spleen, lung and kidney) at different time points. The blood circulation curve (Fig. 3a) depicts that the blood pharmacokinetics of Re NCs obeys the typical two compartment model, and the half-life of the blood distribution phase is calculated to be $2.62 \mathrm{~h}$, indicating a relatively rapid blood elimination in vivo. ${ }^{\mathbf{4 0 , 4 5}}$ Organ biodistribution (Fig. 3b) shows that the Re content in the kidney is as high as $9.15 \pm 3.14 \% \mathrm{ID} / \mathrm{g} 0.5 \mathrm{~h}$ after injection, which is comparable to those of the liver and spleen, suggesting that Re NCs could rapidly arrived at the kidney probably due to their small size and biodegradability. Over time, the Re content in the kidney significantly decreased, which should be attributed to the further renal clearance of Re NCs. In addition, the Re content in the liver and spleen increased at $6 \mathrm{~h}$ and then decreased at $24 \mathrm{~h}$ because of the reticuloendothelial system (RES) and slow biodegradability of exogenous nanoagents in the liver and spleen, indicating that more time was required for complete clearance. ${ }^{\mathbf{4}}$ Such pharmacokinetic and biodistribution behavior of Re NCs is similar to that of many other biodegradable nanoagents including black phosphorus, ${ }^{40}$ porphyrin nanodots, ${ }^{19}$ and $\mathrm{Mo}_{2} \mathrm{C}$ nanosheets. ${ }^{47}$

To evaluate the renal clearance efficiency, PEG-SH modified Au nanoparticles (Au NPs) with a similar size (inset of Fig. 3d) and ammonium perrhenate $\left(\mathrm{NH}_{4} \mathrm{ReO}_{4}\right)$ are used as controls. It is found that about $23 \%$ of Re element is cleared into urine $24 \mathrm{~h}$ after the injection of Re NCs, which is close to the renal clearance efficiency of $\mathrm{NH}_{4} \mathrm{ReO}_{4}$ (25\%) (Fig. 3c and d). In sharp contrast, Au NPs show a much lower renal clearance efficiency, and less than $1 \%$ of $\mathrm{Au}$ element is found in urine, attributed to the inertness and non-biodegradability of Au NPs. Considering the degradation properties of Re NCs into $\mathrm{ReO}_{4}{ }^{-}$ions after exposure of $\mathrm{H}_{2} \mathrm{O}_{2}$, the renal clearance behavior of Re NCs from the body should be similar to that of soluble $\mathrm{ReO}_{4}{ }^{-}$ions,
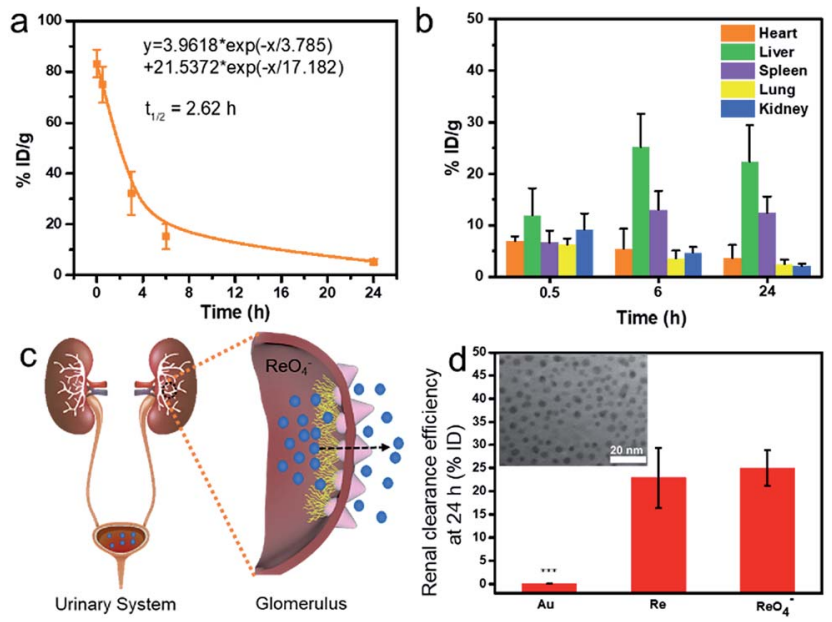

Fig. 3 (a) The blood circulation of and (b) biodistribution of Re NCs in major organs after intravenous injection $\left(0.1 \mathrm{~mL}, 2 \mathrm{mg} \mathrm{mL}^{-1}\right)$ in ICR mice $(n=5)$. (c) Schematic of the renal clearance process of Re NCs; (d) renal clearance efficiencies of Au NPs, Re NCs and $\mathrm{NH}_{4} \mathrm{ReO}_{4} 24 \mathrm{~h}$ after injection $\left(2 \mathrm{mg} \mathrm{mL}^{-1}, 0.2 \mathrm{~mL}\right)$; inset is the TEM image of Au NPs (mean \pm s.d., $* * * p<0.001$ ). suggesting high potential of complete clearance from the body in a reasonable period because ions are commonly more easily cleared from the body than nanoparticles. ${ }^{48-51}$ The effective renal clearance guarantees the safety of Re NCs for biomedical applications.

\section{Cytotoxicity and photothermal ablation in vitro of Re NCs}

The low toxicity of nanomaterials is another vital issue for their potential biomedical applications. To evaluate the cell toxicity of Re NCs, a standard MTT assay is conducted, revealing that almost no toxicity is found when HUVECs are incubated with various concentrations of Re NCs (Fig. 4a). Even when the concentration of Re NCs is as high as 500 ppm, more than $90 \%$ of HUVECs are alive, suggesting the good biocompatibility in vitro of Re NCs. Moreover, MTT results also show the low cytotoxicity of $\mathrm{NH}_{4} \mathrm{ReO}_{4}$ and the products after the oxidization of Re NCs, suggesting the low-toxicity of Re NCs whether before or after oxidization, which is in accordance with the previous report. ${ }^{37}$ In addition, a hemolysis test is conducted to evaluate the blood biocompatibility of Re NCs. The hemolysis rates of Re NCs with different concentrations after incubation in blood for $2 \mathrm{~h}$ are far below the hemolysis rate threshold value of $5 \%$, indicating their excellent blood biocompatibility (Fig. 4b).

Encouraged by the high photothermal performance and extremely low toxicity of Re NCs, photothermal ablation experiments in vitro were conducted. To visualize the localized photothermal killing of cancer cells, 4T1 cells were treated differently. As shown in Fig. 4c, cancer cells can be effectively killed only in the presence of both Re NCs and laser irradiation $(2 \mathrm{~W}, 808 \mathrm{~nm})$ attributed to the strong photothermal effect of Re NCs. Further MTT assay reveals that the killing efficiency is strongly associated with Re NC concentrations, and more cells are killed with the increase of Re NC concentrations (Fig. 4d). Especially, less than $15 \%$ of $4 \mathrm{~T} 1$ cells remained alive when
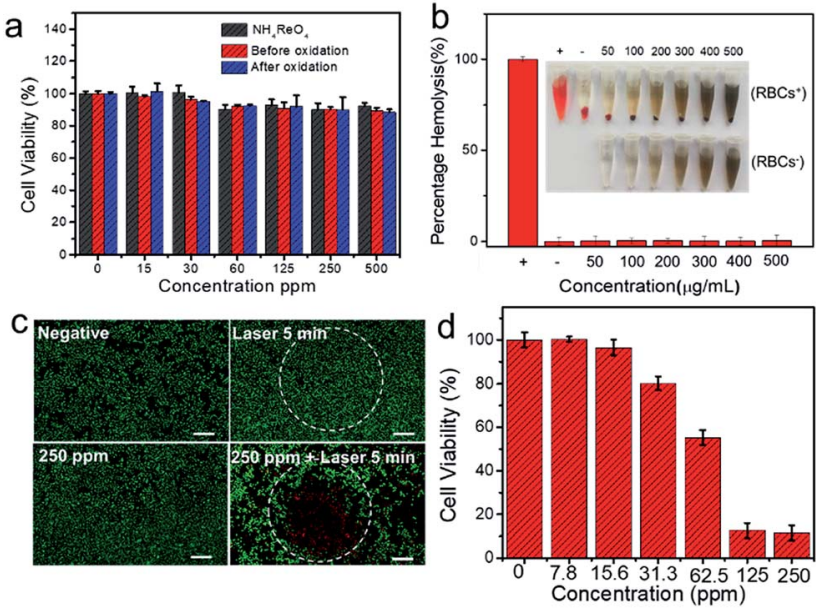

Fig. 4 (a) Cell viability of HUVECs treated with different concentrations of $\mathrm{NH}_{4} \mathrm{ReO}_{4}$, Re NCs and the oxidization products of Re NCs; (b) hemolysis test of different concentrations of Re NCs; (c) fluorescence images of $4 \mathrm{~T} 1$ cells treated with/without a laser or with/without Re NCs. Scale bar: $0.5 \mathrm{~mm}$; (d) concentration-dependent viabilities of 4T1 cells treated with Re NC dispersions under laser irradiation. 
incubated with Re NC dispersions with a concentration of 125 ppm under laser irradiation, indicating the excellent ablation functionality of cancer cells.

\section{Photothermal ablation performance in vivo of Re NCs}

Then, the photothermal ablation in vivo experiment was further conducted. When the tumor volume reaches about $30 \mathrm{~mm}^{3}, 4 \mathrm{~T} 1$ tumor-bearing mice are divided into four groups, i.e. "PBS", "Re", "PBS + NIR" and "Re + NIR". The temperature change of the tumor region of the "Re + NIR" group is $32.3{ }^{\circ} \mathrm{C}$, while only a slightly elevated temperature is observed for the "PBS + NIR" group (Fig. 5a and b). For the treatment group, the tumors are completely ablated, without tumor reoccurrence within 16 days (Fig. 5c). In comparison, a sharp tumor growth for the other three groups is observed (Fig. 5e), and the tumors from different groups are collected as shown in Fig. S8. $\dagger$ After laser irradiation, the tumor slices were stained with hematoxylin-eosin (H\&E) to investigate the antitumor activity at the cellular level. Broken, smaller or fewer nuclei in the illumination region are observed while no obvious destruction is observed for the other three groups (Fig. 5d). The survival rate of mice after various treatments also depicts a high therapeutic efficiency of Re NCs for 4T1 tumors with the assistance of laser irradiation (Fig. S9†). After PTT treatments, major organs (heart, liver, spleen, lung and kidney) of mice from different groups are extracted, and no visible tissue damage or inflammation is observed (Fig. S10 and S11†), suggesting that Re NCs are not toxic to normal organs. In addition, no obvious body weight change is observed in all

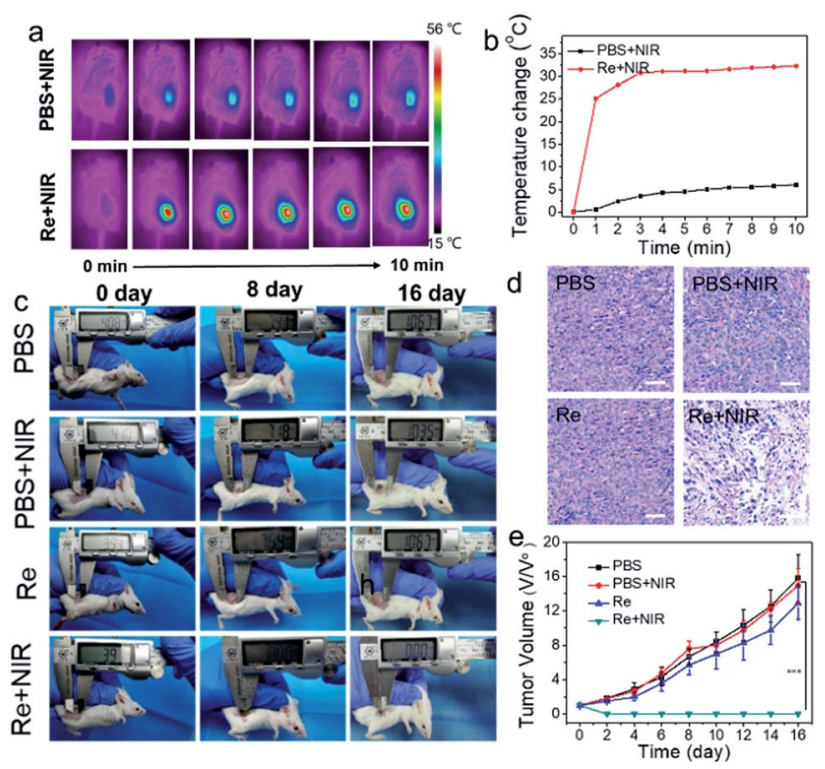

Fig. 5 Photothermal therapy of 4T1 in vivo. (a) Thermal imaging of tumor-bearing mice after injection of PBS or Re NCs $(25 \mu \mathrm{L}, 2 \mathrm{mg}$ $\mathrm{mL}^{-1}$ ); (b) temperature change of the tumor section upon laser irradiation for $10 \mathrm{~min}\left(1 \mathrm{~W} \mathrm{~cm} \mathrm{~cm}^{-2}, 808 \mathrm{~nm}\right)$; (c) digital photographs of 4T1bearing BALB/c mice from different groups at different times $(0,8$ and 16 days); (d) H\&E images of tumor sections harvested from the mice. Scale bar is $50 \mu \mathrm{m}$; (e) tumor volume change during treatment $(n=5$, mean \pm s.d., $* * * p<0.001$ ).

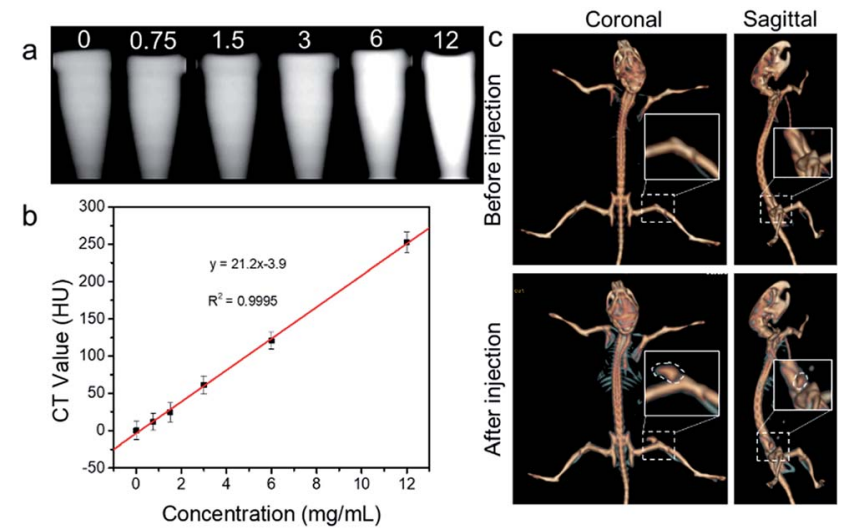

Fig. 6 (a) CT images in vitro of Re NC dispersions at different concentrations as indicated. (b) The corresponding CT value of Re NC dispersions; (c) CT imaging in vivo of $4 \mathrm{~T} 1$ tumor-bearing BALB/c mice before and after injection. The tumor region is marked by a white dotted line $(n=5)$.

groups, indicating the low in vivo toxicity of Re NCs (Fig. S12†). Hence, Re NCs can perform as high effective photothermal agents for the ablation of tumors.

\section{In vitro and in vivo CT imaging of Re NCs}

Apart from the role as degradable photothermal agents, Re NCs may also be used as high-performance CT contrast agents due to the high atomic number of $\operatorname{Re}(Z=75)$ element. Although numerous nanoparticles containing a high- $Z$ element including $\mathrm{TaO}_{x}, \mathrm{WS}_{2}$ and $\mathrm{Bi}_{2} \mathrm{~S}_{3}$ have been reported as an excellent alternative to clinical iodine-based small-molecule agents, the nonbiodegradation and limited renal clearance may harm their further clinical applications. ${ }^{52-54}$ Hence, the as-prepared Re NCs with both biodegradation and renal clearance properties may have higher potential for clinical CT contrast. As shown in Fig. $6 a$ and b, progressively brighter images of Re NC dispersions emerged with the increase of nanocluster concentrations under a clinical CT scanner, and the X-ray absorption coefficient of Re NCs is determined to be as high as 21.2 Hounsfield units (HU) $\mathrm{mL} \mathrm{mg}^{-1}$, higher than that of commercial iopromide (15.9 $\mathrm{HU} \mathrm{mL} \mathrm{mg^{-1 }}$ ) in clinics. ${ }^{55}$ To further demonstrate the CT enhanced ability of Re NCs, a CT imaging experiment in vivo was conducted. The tumor (marked by a white dotted line) contrast could be obviously detected after intratumoral injection of Re NCs $\left(50 \mu \mathrm{L}, 20 \mathrm{mg} \mathrm{mL}^{-1}\right)$ while no tumor signal is found before injection under the same conditions, indicating the excellent contrast ability of Re NCs (Fig. 6c). Considering the excellent photothermal performance, Re NCs have high potential as degradable metallic theranostic agents for CT imagingguided photothermal therapy.

\section{Conclusions}

In conclusion, PEGylated rhenium NCs have been successfully fabricated via a simple and quick liquid-phase reduction method, and their high potential as a degradable and renalclearable agent for the ablation of $4 \mathrm{~T} 1$ tumors has been 
revealed and demonstrated for the first time. The obtained $\mathrm{Re}$ NCs are $\mathrm{H}_{2} \mathrm{O}_{2}$-sensitive degradable into biocompatible $\mathrm{ReO}_{4}{ }^{-}$ ions, ensuring the potential of complete clearance. In addition, Re NCs show a higher X-ray absorption coefficient than commercial iopromide in clinics. This is the first report about degradable noble metal nanoparticles for both cancer imaging and therapy. The unique features of high photothermal effects, obvious CT contrast, $\mathrm{H}_{2} \mathrm{O}_{2}$-trigged degradability and effective renal clearance promise that Re NCs as multifunctional metal agents have high potential for clinical translation.

\section{Conflicts of interest}

There are no conflicts to declare.

\section{Acknowledgements}

This work is supported by the National Natural Science Foundation of China (No. 31800834; 51572067; 21501039), the State Key Lab of Advanced Welding and Joining (No. AWJ-19M12), the Fundamental Research Funds for the Central Universities (No. JZ2018HGTA0202; JZ2018HGPA0273; JZ2018HGTB0247; JZ2018HGBZ0156; JZ2018HGPA0269), and the Anhui Provincial Natural Science Foundation (No. JZ2015AKZR0642; 1608085MH188; 1708085ME114).

\section{References}

1 F. Liu, X. He, H. Chen, J. Zhang, H. Zhang and Z. Wang, Nat. Commun., 2015, 6, 8003.

2 C. Zhang, W. Bu, D. Ni, C. Zuo, C. Cheng, Q. Li, L. Zhang, Z. Wang and J. Shi, J. Am. Chem. Soc., 2016, 138, 8156-8164.

3 Z. Tao, X. Dang, X. Huang, M. D. Muzumdar, E. S. Xu, N. M. Bardhan, H. Song, R. Qi, Y. Yu, T. Li, W. Wei, J. Wyckoff, M. J. Birrer, A. M. Belcher and P. P. Ghoroghchian, Biomaterials, 2017, 134, 202-215.

4 J. Wang and G. Liu, Angew. Chem., Int. Ed., 2018, 57, 30083010.

5 X. Hu, J. Sun, F. Li, R. Li, J. Wu, J. He, N. Wang, J. Liu, S. Wang, F. Zhou, X. Sun, D. Kim, T. Hyeon and D. Ling, Nano Lett., 2018, 18, 1196-1204.

6 Y. Zhao, J. Peng, J. Li, L. Huang, J. Yang, K. Huang, H. Li, N. Jiang, S. Zheng, X. Zhang, Y. Niu and G. Han, Nano Lett., 2017, 17, 4096-4100.

7 M. Zhou, J. Li, S. Liang, A. K. Sood, D. Liang and C. Li, ACS Nano, 2015, 9, 7085-7096.

8 P. Lei, R. An, P. Zhang, S. Yao, S. Song, L. Dong, X. Xu, K. Du, J. Feng and H. Zhang, Adv. Funct. Mater., 2017, 27, 1702018. 9 Y. a. Tang, T. Yang, Q. Wang, X. Lv, X. Song, H. Ke, Z. Guo, X. Huang, J. Hu, Z. Li, P. Yang, X. Yang and H. Chen, Biomaterials, 2018, 154, 248-260.

10 X. Zhu, J. Li, X. Qiu, Y. Liu, W. Feng and F. Li, Nat. Commun., 2018, 9, 2176.

11 J. Conde, N. Oliva, Y. Zhang and N. Artzi, Nat. Mater., 2016, 15, 1128.

12 Y. Chen, L. Cheng, Z. Dong, Y. Chao, H. Lei, H. Zhao, J. Wang and Z. Liu, Angew. Chem., Int. Ed., 2017, 56, 12991-12996.
13 Q. Chen, C. Liang, X. Sun, J. Chen, Z. Yang, H. Zhao, L. Feng and Z. Liu, Proc. Natl. Acad. Sci. U. S. A., 2017, 114(21), 53435348.

14 J. F. Lovell, C. S. Jin, E. Huynh, H. Jin, C. Kim, J. L. Rubinstein, W. C. W. Chan, W. Cao, L. V. Wang and G. Zheng, Nat. Mater., 2011, 10, 324.

15 S. Wang, Y. Chen, X. Li, W. Gao, L. Zhang, J. Liu, Y. Zheng, H. Chen and J. Shi, Adv. Mater., 2015, 27, 7117-7122.

16 W. Zhang, G. Deng, B. Li, X. Zhao, T. Ji, G. Song, Z. Xiao, Q. Cao, J. Xiao, X. Huang, G. Guan, R. Zou, X. Lu and J. $\mathrm{Hu}$, Biomaterials, 2018, 159, 68-81.

17 S. Zhang, C. Sun, J. Zeng, Q. Sun, G. Wang, Y. Wang, Y. Wu, S. Dou, M. Gao and Z. Li, Adv. Mater., 2016, 28, 8927-8936.

18 J. Mou, P. Li, C. Liu, H. Xu, L. Song, J. Wang, K. Zhang, Y. Chen, J. Shi and H. Chen, Small, 2015, 11, 2275-2283.

19 Q. Zou, M. Abbas, L. Zhao, S. Li, G. Shen and X. Yan, J. Am. Chem. Soc., 2017, 139, 1921-1927.

20 Z. Sun, H. Xie, S. Tang, X. F. Yu, Z. Guo, J. Shao, H. Zhang, H. Huang, H. Wang and K. Chu Paul, Angew. Chem., Int. Ed., 2015, 54, 11526-11530.

21 T. Zhang, Y. Wan, H. Xie, Y. Mu, P. Du, D. Wang, X. Wu, H. Ji and L. Wan, J. Am. Chem. Soc., 2018, 140(24), 7561-7567.

22 H. Wang, X. Yang, W. Shao, S. Chen, J. Xie, X. Zhang, J. Wang and Y. Xie, J. Am. Chem. Soc., 2015, 137, 11376-11382.

23 W. Chen, J. Ouyang, H. Liu, M. Chen, K. Zeng, J. Sheng, Z. Liu, Y. Han, L. Wang, J. Li, L. Deng, Y. N. Liu and S. Guo, Adv. Mater., 2016, 29, 1603864.

24 M. Qiu, D. Wang, W. Liang, L. Liu, Y. Zhang, X. Chen, D. K. Sang, C. Xing, Z. Li, B. Dong, F. Xing, D. Fan, S. Bao, H. Zhang and Y. Cao, Proc. Natl. Acad. Sci. U. S. A., 2018, 115, 501-506.

25 L. R. Hirsch, R. J. Stafford, J. A. Bankson, S. R. Sershen, B. Rivera, R. E. Price, J. D. Hazle, N. J. Halas and J. L. West, Proc. Natl. Acad. Sci. U. S. A., 2003, 100, 1354913554.

26 X. Huang, I. H. El-Sayed, W. Qian and M. A. El-Sayed, J. Am. Chem. Soc., 2006, 128, 2115-2120.

27 A. F. Bagley, S. Hill, G. S. Rogers and S. N. Bhatia, ACS Nano, 2013, 7, 8089-8097.

28 X. Huang, S. Tang, X. Mu, Y. Dai, G. Chen, Z. Zhou, F. Ruan, Z. Yang and N. Zheng, Nat. Nanotechnol., 2010, 6, 28.

29 P. Cherukuri, E. S. Glazer and S. A. Curley, Adv. Drug Delivery Rev., 2010, 62, 339-345.

30 F. Chen and W. Cai, Nanomedicine, 2015, 10, 1-3.

31 J. V. Rojas and C. H. Castano, Radiat. Phys. Chem., 2014, 99, 1-5.

32 J. Bedia, L. Calvo, J. Lemus, A. Quintanilla, J. A. Casas, A. F. Mohedano, J. A. Zazo, J. J. Rodriguez and M. A. Gilarranz, Colloids Surf., A, 2015, 469, 202-210.

33 J. Yi, J. T. Miller, D. Y. Zemlyanov, R. Zhang, P. J. Dietrich, F. H. Ribeiro, S. Suslov and M. M. Abu-Omar, Angew. Chem., Int. Ed., 2014, 53, 833-836.

34 J. M. Jeong and J.-K. Chung, Cancer Biother.Radiopharm., 2003, 18, 707-717.

35 D. Séhédic, I. Chourpa, C. Tétaud, A. Griveau, C. Loussouarn, S. Avril, C. Legendre, N. Lepareur, D. Wion, F. Hindré, 
F. Davodeau and E. Garcion, Theranostics, 2017, 7, 45174536.

36 Z.-H. Miao, L.-X. Lv, K. Li, P.-Y. Liu, Z. Li, H. Yang, Q. Zhao, M. Chang, L. Zhen and C.-Y. Xu, Small, 2018, 14, 1703789.

37 T. J. Haley and F. D. Cartwright, J. Pharm. Sci., 1968, 57, 321323.

38 T. P. Szatrowski and C. F. Nathan, Cancer Res., 1991, 51, 794798.

39 G. Yang, L. Xu, Y. Chao, J. Xu, X. Sun, Y. Wu, R. Peng and Z. Liu, Nat. Commun., 2017, 8, 902.

40 J. Shao, H. Xie, H. Huang, Z. Li, Z. Sun, Y. Xu, Q. Xiao, X.-F. Yu, Y. Zhao, H. Zhang, H. Wang and P. K. Chu, Nat. Commun., 2016, 7, 12967.

41 B. Varnholt, P. Oulevey, S. Luber, C. Kumara, A. Dass and T. Bürgi, J. Phys. Chem. C, 2014, 118, 9604-9611.

42 S. Kundu, L. Ma, W. Dai, Y. Chen, A. M. Sinyukov and H. Liang, ACS Sustainable Chem. Eng., 2017, 5, 10186-10198. 43 H. Huang, R. Hernandez, J. Geng, H. Sun, W. Song, F. Chen, S. A. Graves, R. J. Nickles, C. Cheng, W. Cai and J. F. Lovell, Biomaterials, 2016, 76, 25-32.

44 X. Qian, Z. Gu and Y. Chen, Mater. Horiz., 2017, 4, 800-816. 45 H. Lin, S. Gao, C. Dai, Y. Chen and J. Shi, J. Am. Chem. Soc., 2017, 139, 16235-16247.
46 Q. Jiang, Z. Luo, Y. Men, P. Yang, H. Peng, R. Guo, Y. Tian, Z. Pang and W. Yang, Biomaterials, 2017, 143, 29-45.

47 W. Feng, R. Wang, Y. Zhou, L. Ding, X. Gao, B. Zhou, P. Hu and Y. Chen, Adv. Funct. Mater., 2019, 1901942.

48 E. B. Ehlerding, F. Chen and W. Cai, Adv. Sci., 2016, 3, 1500223.

49 J. G. Croissant, Y. Fatieiev and N. M. Khashab, Adv. Mater., 2017, 29, 1604634.

50 B. Du, X. Jiang, A. Das, Q. Zhou, M. Yu, R. Jin and J. Zheng, Nat. Nanotechnol., 2017, 12, 1096.

51 Q. Wei, Y. Chen, X. Ma, J. Ji, Y. Qiao, B. Zhou, F. Ma, D. Ling, H. Zhang, M. Tian, J. Tian and M. Zhou, Adv. Funct. Mater., 2017, 28, 1704634.

52 Y. Jin, Y. Li, X. Ma, Z. Zha, L. Shi, J. Tian and Z. Dai, Biomaterials, 2014, 35, 5795-5804.

53 L. Cheng, J. Liu, X. Gu, H. Gong, X. Shi, T. Liu, C. Wang, X. Wang, G. Liu, H. Xing, W. Bu, B. Sun and Z. Liu, Adv. Mater., 2013, 26, 1886-1893.

54 K. Ai, Y. Liu, J. Liu, Q. Yuan, Y. He and L. Lu, Adv. Mater., 2011, 23, 4886-4891.

55 A. Xia, M. Chen, Y. Gao, D. Wu, W. Feng and F. Li, Biomaterials, 2012, 33, 5394-5405. 